\title{
Top-of-atmosphere albedo bias from neglecting three-dimensional radiative transfer through clouds
}

\author{
Clare E. Singer ${ }^{1}$, Ignacio Lopez-Gomez ${ }^{1}$, Xiyue Zhang ${ }^{1 *}$, Tapio Schneider ${ }^{1,2}$
}

${ }^{1}$ Department of Environmental Science and Engineering, California Institute of Technology, Pasadena, California, USA.

${ }^{2}$ Jet Propulsion Laboratory, California Institute of Technology, Pasadena, California, USA.

\section{Key Points:}

- One-dimensional radiative treatment of clouds results in a top-of-atmosphere reflected flux bias

- The bias varies with solar zenith angle and cloud type and has clear spatial and seasonal patterns

- The magnitude of the annual- and global-mean flux bias is estimated as $1.6 \mathrm{~W} \mathrm{~m}^{-2}$

*Current affiliation: National Center for Atmospheric Research, Boulder, CO, USA.

Corresponding author: Clare E. Singer, csinger@caltech.edu 


\section{Abstract}

Clouds cover on average nearly $70 \%$ of Earth's surface and are important for the global albedo. The magnitude of the shortwave reflection by clouds depends on their location, optical properties, and 3D structure. Earth system models are unable to perform 3D radiative transfer calculations and thus partially neglect the effect of cloud morphology on albedo. We show how the resulting radiative flux bias depends on cloud morphology and solar zenith angle. Using large-eddy simulations to produce 3D cloud fields, a Monte Carlo code for 3D radiative transfer, and observations of cloud climatology, we estimate the effect of this flux bias on global climate. The flux bias is largest at small zenith angles and for deeper clouds, while the albedo bias is largest (and negative) for large zenith angles. Globally, the radiative flux bias is estimated to be $1.6 \mathrm{~W} \mathrm{~m}^{-2}$ and locally can be on the order of $5 \mathrm{~W} \mathrm{~m}^{-2}$.

\section{Plain Language Summary}

Clouds cover on average about $70 \%$ of Earth's surface and are important for regulating the surface temperature by reflecting nearly $15 \%$ of the incoming energy from the sun back to space. How individual clouds reflect this incoming radiation depends on where they are and what they look like. Earth system models cannot resolve the radiative effect of the detailed morphology of clouds due to computational constraints. Instead, models approximate the way that clouds reflect light, which leads to a bias in the amount of energy reflected back to space. In this study, the reflection bias from neglecting the detailed 3D structure of clouds in radiative transfer calculations is studied to estimate its net effect on climate. It is found that deep thunderstorm clouds near the equator lead to significant biases, due to both their location and size. Globally, the implied energy bias is of a similar magnitude as the energy imbalance created by anthropogenic greenhouse gases. It is important to correct this bias in climate models.

\section{Introduction}

Earth's average albedo is roughly $29 \%$, with clouds accounting for about half of the reflection of solar radiative energy fluxes back to space (Stephens et al., 2015). Accurately simulating clouds is crucial for modeling Earth's albedo. However, Earth system models (ESMs) struggle to accurately represent the mean albedo, its spatial patterns, and its seasonal variability (Bender et al., 2006; Voigt et al., 2013). Simulating clouds 
is difficult for several reasons, but one major factor is their wide range of spatial scales. Clouds have complex three-dimensional morphologies created by turbulent motions at length scales down to tens of meters or smaller. However, the typical resolution of an ESM is around only $10-100 \mathrm{~km}$ in the horizontal and $100-200 \mathrm{~m}$ in the vertical in the lower troposphere (Schneider et al., 2017). This discrepancy means that clouds are not explicitly resolved in ESMs. Instead, they are represented by parameterizations and, for purposes of radiative transfer (RT) calculations, are approximated as plane-parallel structures within grid cells (Marshak \& Davis, 2005). Semi-empirical parameterizations account for heterogeneity of optical properties on subgrid-scales (e.g., Macke et al., 1999; Wood et al., 2005; Gimeno García et al., 2012).

ESMs resort to two main simplifications when performing RT calculations: (1) the plane-parallel approximation (PPA) made on the cloud morphology, which assumes clouds are smeared out across the entire grid box, and (2) the independent-pixel approximation (IPA), which assumes no horizontal radiative fluxes between neighboring grid cells. These different approximations amount to either ignoring the horizontal heterogeneity of cloud optical properties or considering the heterogeneity in optical properties, but assuming a net zero transfer of photons, respectively (R. Cahalan \& Wiscombe, 1992; R. F. Cahalan et al., 1994). The PPA is a consequence of the limited spatial resolution of climate models, while the IPA is necessary to make radiative transfer calculations tractable.

Yet, the importance of the structure of clouds on radiative transfer has been recognized for nearly 50 years (e.g., McKee \& Cox, 1974; Barker, 1994) and has recently received renewed attention since advances in computation allow more direct simulation of 3D RT (e.g., Emde et al., 2016; Schäfer et al., 2016; Villefranque et al., 2019). For example, one topic that has garnered particular interest in the literature is the effect of broken cloud fields (Barker, 1994; Hinkelman et al., 2007; Gristey et al., 2019), which considers the subgrid-scale heterogeneity in liquid water path; however, it does not consider the effects of 3D optics. Veerman et al. (2020) show the importance of including the 3D optical effects (or the bias resulting from the IPA) on the dynamics of shallow cumulus clouds and the coupling between the boundary layer and land surface.

The PPA may be avoided in ESMs using embedded 2D cloud-resolving models (Kooperman et al., 2016), an approach known as cloud superparameterization (Khairoutdinov \& Randall, 2001). However, 3D radiation computations will remain too expensive to run in ESMs 
in the near future, making simplifications such as the IPA necessary. The structural differences between IPA and a full three-dimensional RT calculation have been documented before (Barker et al., 2003; Marshak, Davis, Wiscombe, \& Titov, 1995; Barker et al., 2012), and many alternatives to IPA have been proposed to minimize their mismatch (Marshak, Davis, Wiscombe, \& Cahalan, 1995; Várnai \& Davies, 1999; Frame et al., 2009; Hogan \& Shonk, 2013; Wissmeier et al., 2013; Okata et al., 2017). Nevertheless, most studies have been focused on theoretical cases, small spatial and temporal domains, or improving satellite retrieval algorithms. Some notable exceptions are Cole et al. (2005) and Barker et al. (2015), who compared 3D and IPA RT calculations to estimate the bias present in ESMs using a superparameterized cloud resolving model and coarse-resolution, twodimensional cloud fields retrieved from CloudSAT and CALIPSO, respectively.

Here we discuss the magnitude of the bias that results from making the IPA during radiative transfer calculations in global climate simulations. We use large-eddy simulations (LES) to generate three-dimensional cloud fields representing three canonical cloud regimes: shallow convection, stratocumulus, and deep convection. Then we calculate the bias between the true reflected flux and the flux approximated by IPA using a Monte Carlo RT code. The radiative flux bias is shown to vary with zenith angle and cloud type. Because the zenith angle varies with the diurnal and seasonal cycle, we quantify the effect of the 3D bias on these timescales. Finally, the 3D flux bias is mapped onto observations of cloud climatology to estimate the global and spatial effect on climate simulations where three-dimensional radiative fluxes are neglected. As stated earlier, most ESMs make both the IPA and some variant of the PPA for radiative transfer calculations, so the bias associated with the IPA is an underestimate of the total bias. However, because of the diversity of assumptions made by global models to account for phenomena such as cloud overlap, and the fundamental resolution dependence of the PPA, in this study we focus on the bias resulting from RT using only the IPA on fully resolved 3D cloud structures from LES.

\section{Methods}

\subsection{Large-eddy simulations of clouds}

Three-dimensional cloud fields are generated from high-resolution LES using the anelastic solver PyCLES (Pressel et al., 2015, 2017). The LES are run in three dynam- 
ical regimes to simulate shallow cumulus ( $\mathrm{ShCu}$ ), stratocumulus ( $\mathrm{Sc}$ ), and deep-convective clouds $(\mathrm{Cb})$; details can be found in the Supporting Information. ShCu clouds are convective clouds with typical cloud cover of $10-20 \%$ and cloud top height (CTH) around $2 \mathrm{~km}$. They occur frequently over low- and mid-latitude oceans. In this study, $\mathrm{ShCu}$ are represented by two LES case studies, BOMEX and RICO, which represent non-precipitating and precipitating convection over tropical oceans, respectively (Siebesma et al., 2003; vanZanten et al., 2011). Sc clouds are shallow, with CTH only around $1 \mathrm{~km}$, but optically thick for longwave radiation. They have cloud cover near $100 \%$ and typically blanket subtropical oceans off the west coasts of continents. Sc are represented by the DYCOMSII RF01 LES case of a Sc deck off the coast of California (Stevens et al., 2005). Cb clouds are deep convective thunderstorm clouds that occur frequently over midlatitude continents in summer and at low latitudes, e.g., in the intertropical convergence zone (ITCZ). Their CTH can reach up to $15 \mathrm{~km}$ or higher, they often contain ice, and anvils at the top contribute to a cloud cover around $30 \%$. Cb clouds are represented in this paper by the TRMM-LBA LES case based on measurements of convection over land in the Amazon (Grabowski et al., 2006).

An ensemble of snapshots is used to estimate the mean and variance of the bias for each cloud type. The ensemble sizes were chosen to capture the natural variability of morphology in each LES case: 10 for ShCu (BOMEX and RICO), 5 for Sc (DYCOMSII RF01, and 15 for $\mathrm{Cb}$ (TRMM-LBA). For $\mathrm{ShCu}$ and $\mathrm{Sc}$ we take snapshots evenly spaced in time starting once the simulation has reached a statistically steady-state, after an initial spin-up period. The snapshots are chosen to be at least one convective turnover time apart (1 hour for BOMEX and RICO and 30 minutes for DYCOMS-II RF01. For the $\mathrm{Cb}$ case we take snapshots from an initial-condition ensemble at a time point representative of deep convection, characterized by stable liquid and ice water paths, occurring at 13:00 local time in the TRMM-LBA simulation. All subsequent results are calculated as the mean over the ensemble of cloud field snapshots.

\subsection{Radiative transfer computations}

The RT calculations were done using the libRadtran software package with the MYSTIC Monte Carlo solver (Mayer \& Kylling, 2005; Mayer, 2009; Emde et al., 2016). The MYSTIC solver requires, as input, three-dimensional fields of liquid/ice water content and particle effective radius. The LES uses bulk microphysics schemes (2-moment for 
liquid, 1-moment for ice) and does not explicitly compute the effective radius. To compute the effective radius, we follow the parameterization from Ackerman et al. (2009) and Blossey et al. (2013) for liquid and Wyser (1998) for ice (Supporting Information). For the RT, MYSTIC computes the scattering phase function. In the case of liquid droplets, which can be assumed spherical, the full Mie phase function is used. For the case of ice clouds, a parameterization of the habit-dependent scattering must be used. We find that the results are insensitive to the choice of ice parameterization (Supporting Information), mostly because the reflected flux signal is dominated by the liquid phase for the cloud types simulated.

\section{Results and Discussions}

\subsection{Radiative flux bias dependence on zenith angle}

The radiative flux bias is measured (in $\mathrm{W} \mathrm{m}^{-2}$ ) as the difference in reflected irradiance between the 1D and 3D RT calculations. A positive bias means that the 1D is reflecting more energy than the 3D truth, and the Earth system is artificially dimmed. The albedo bias $(\Delta \alpha)$ is computed as the flux bias $(\Delta F)$ divided by the total incoming solar flux $\left(F_{\text {in }}\right)$,

$$
\Delta \alpha=\frac{\Delta F}{F_{\text {in }}} \times 100 \%
$$

Figure 1 shows the flux and albedo biases (1D-3D) for the four cases of $\mathrm{ShCu}, \mathrm{Sc}$, and $\mathrm{Cb}$ clouds. Shown are both the flux and albedo biases resulting from RT computations using the IPA and also RT using the horizontally averaged cloud fields (PPA). We do not try to account for cloud overlap (e.g. Tompkins \& Di Giuseppe, 2007, 2015) or resolution dependence (e.g. Oreopoulos \& Davies, 1998) in the PPA computations, so this bias may be regarded as an upper bound for biases present in ESMs.

For all cloud types, the bias from the PPA is larger than from the IPA (note the different y-axes between the left and right columns in Figure 1). Sc show negligible deviation between 1D and 3D reflected fluxes, especially for the IPA. For the PPA the bias from all cloud types is always positive, meaning the PPA always overestimates the amount of reflected radiation. For convective clouds ( $\mathrm{ShCu}$ and $\mathrm{Cb}$ ), the bias from the IPA is positive, except for $\mathrm{ShCu}$ at very large solar zenith angles. ShCu scatter far fewer photons than $\mathrm{Cb}$ due to the low cloud cover and their small vertical extent $(2-3 \mathrm{~km}) . \mathrm{Cb}$ exhibit both the largest reflected irradiance and also the largest bias between the 1D (IPA 

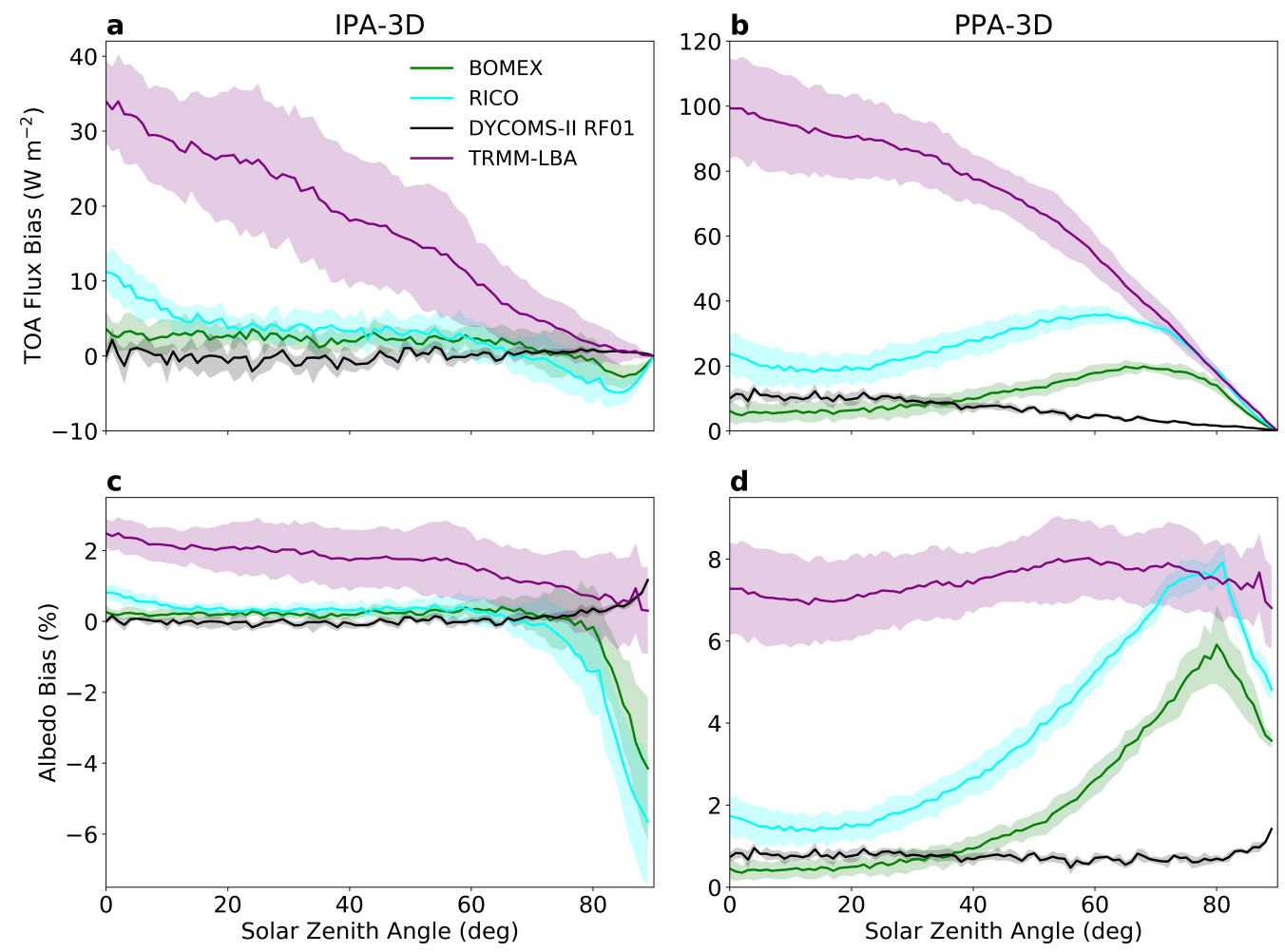

Figure 1. Bias (1D-3D) in TOA reflected flux (a, b) and albedo (c, d) as a function of zenith angle for ShCu (BOMEX and RICO), Sc (DYCOMS-II RF01), and Cb (TRMM-LBA). The left column (a, c) shows the bias resulting from the IPA, and the right column (b, d) the bias resulting from the PPA. For each cloud type, average fluxes (with shaded $1 \sigma$ error bars) are computed over the individual snapshots. Positive bias means the $1 \mathrm{D}$ approximation is reflecting more incoming flux than in the 3D RT calculation. 
and PPA) and 3D RT calculations. The convective clouds show much more variation than the stratiform clouds between snapshots due to the variability in cloud cover even in a statistically steady state. Cb show nearly uniform albedo bias across zenith angles for the PPA. At large zenith angles, $\mathrm{ShCu}$ show a negative flux and albedo bias for IPA, but a large, positive flux and albedo bias for PPA.

In the IPA, the horizontal photon fluxes are ignored. For the Sc clouds that uniformly cover the whole domain (Figure 2), this assumption has little effect: the flux bias is near zero for all zenith angles. For very small zenith angles, when the sun is overhead, the convective clouds ( $\mathrm{ShCu}$ and $\mathrm{Cb}$ ) produce a positive flux and albedo bias, meaning that the IPA overestimates the scattering. This is due to the fact that the IPA overestimates the path length of a photon through the cloud; in reality (3D) the photons have a higher chance to exit the cloud through the sides (Schäfer et al., 2016). For large zenith angles $\left(>70^{\circ}\right)$, the flux and albedo bias from $\mathrm{ShCu}$ is negative. This is because, at these large zenith angles, the $\mathrm{ShCu}$ begin to shadow each other, and scattering from the sides of the clouds becomes dominant. This "shadowing effect" has been discussed extensively in the literature (e.g. Marshak \& Davis, 2005; Frame et al., 2009; Gristey et al., 2019); for example, Veerman et al. (2020) show the importance of coupling between the shadowing and surface fluxes for cloud dynamics. In the IPA, when the horizontal fluxes are ignored, the cloud sides are not exposed, and the scattering is underestimated. These effects can be understood from Figure 2, which shows illustrations of the clouds from the four LES cases.

For the rest of the discussion, 1D RT refers only to the IPA on the fully resolved $3 \mathrm{D}$ clouds; it does not include the horizontal homogenization (PPA).

\subsection{Seasonal cycle of radiative flux bias}

To assess the climate impact of the radiation bias resulting from the IPA, we consider the flux and albedo bias for each cloud type as a function of day of year and latitude. This calculation is done by assuming that the LES-generated cloud field is present at any given latitude circle on any given day of the year. This exercise is done not to be realistic, but to demonstrate the impact each cloud type might have on Earth. For any location and time, including a diurnal cycle, the solar zenith angle is calculated and the flux bias is estimated based on the results presented in Figure 1. The flux bias is com- 
a

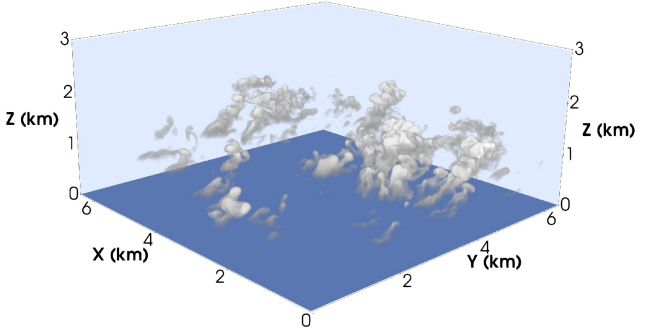

C

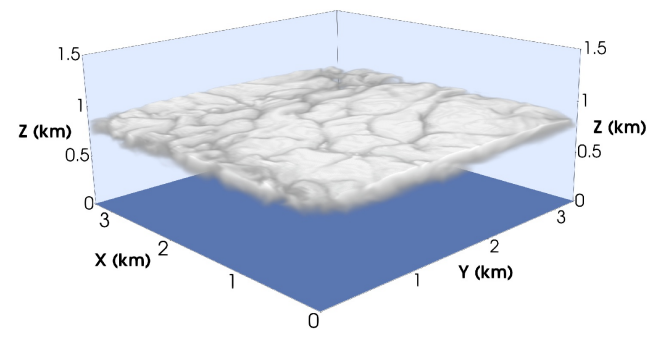

b $\quad \mathrm{RICO}$

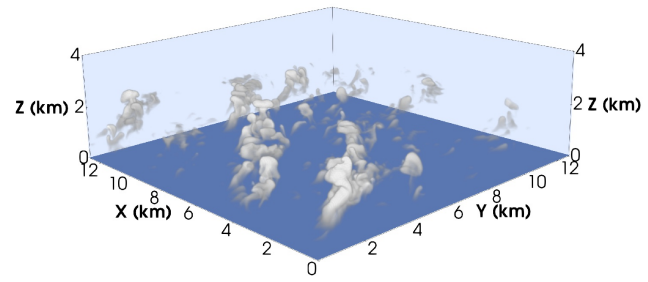

d TRMM-LBA

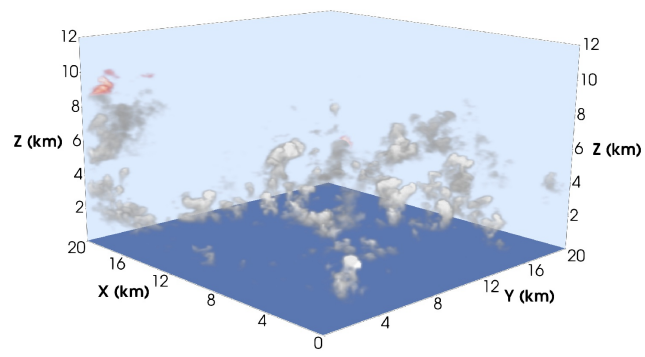

Figure 2. Snapshots of LES clouds, showing liquid water specific humidity (gray to white, low to high) and ice water specific humidity (red to white, low to high). (a) and (b) Shallow convective clouds. (c) Stratocumulus clouds. (d) Deep convective clouds. Note that the domain sizes vary between the cases. At high zenith angles, cloud shadowing becomes important for $\mathrm{ShCu}$ because the individual clouds can shadow a large portion of the domain and scattering from the cloud sides becomes dominant due to the low angle of the incoming photons. 
puted hourly and averaged to show the diurnal-mean bias. The albedo bias is computed analogously.

Figure 3 shows the TOA flux and albedo biases for each cloud type as a function of day of year and latitude. The annual mean bias is shown in the insets to the left of each panel. All cloud types show zero flux bias in regions of polar night where there is no incoming solar flux. Both $\mathrm{ShCu}$ cases show similar patterns of flux bias with latitude and time. As seen in Figure 1, these cases both have a negative bias for high solar zenith angles $\left(>70^{\circ}\right.$ ), and therefore the net flux (and albedo) bias during the shoulder seasons at very high latitudes is negative. At lower latitudes, where the diurnally averaged zenith angle is never larger than $70^{\circ}$, the net flux bias is always positive. Sc show a very small flux (and albedo) bias for all zenith angles due to their high cloud cover and optical depth, but they do exhibit a small positive flux bias $\left(\sim 0.5 \mathrm{~W} \mathrm{~m}^{-2}\right)$ during summer in high latitudes. For $\mathrm{Cb}$, the flux bias is very large, always positive, and varies roughly linearly with zenith angle (Figure 1). This gives rise to a bias pattern that roughly mimics the insolation pattern with latitude and day of year. The albedo bias for $\mathrm{Cb}$ is largest and positive in the high-latitudes during summer because the mean zenith angle is small, since the sun never sets.

In addition to the diurnal bias that arises from changes in zenith angle from sunrise to sunset over the course of the day, there is a seasonal cycle in the radiation bias resulting from Earth's orbital obliquity. For instance, equatorial deep convective clouds create a TOA albedo bias that peaks during northern hemisphere summer and has a minimum in winter.

\subsection{Implications for Climate Models}

To make an assessment of the effect that the 3D radiative transfer through cloud fields has on climate simulated with ESMs, we must account for the climatological occurrence of different cloud types in space and time. We observe a strong positive correlation between CTH and flux bias (Figure 4). Sc have the lowest cloud top and smallest flux bias, and $\mathrm{Cb}$ have the highest.

We regress the flux bias against CTH for 91 evenly spaced solar zenith angles between 0 and $90^{\circ}$, constraining the regression lines to pass through the origin because there is no flux bias in clear-sky conditions $(\mathrm{CTH}=0)$. We define the $\mathrm{CTH}$ to be the 90th 

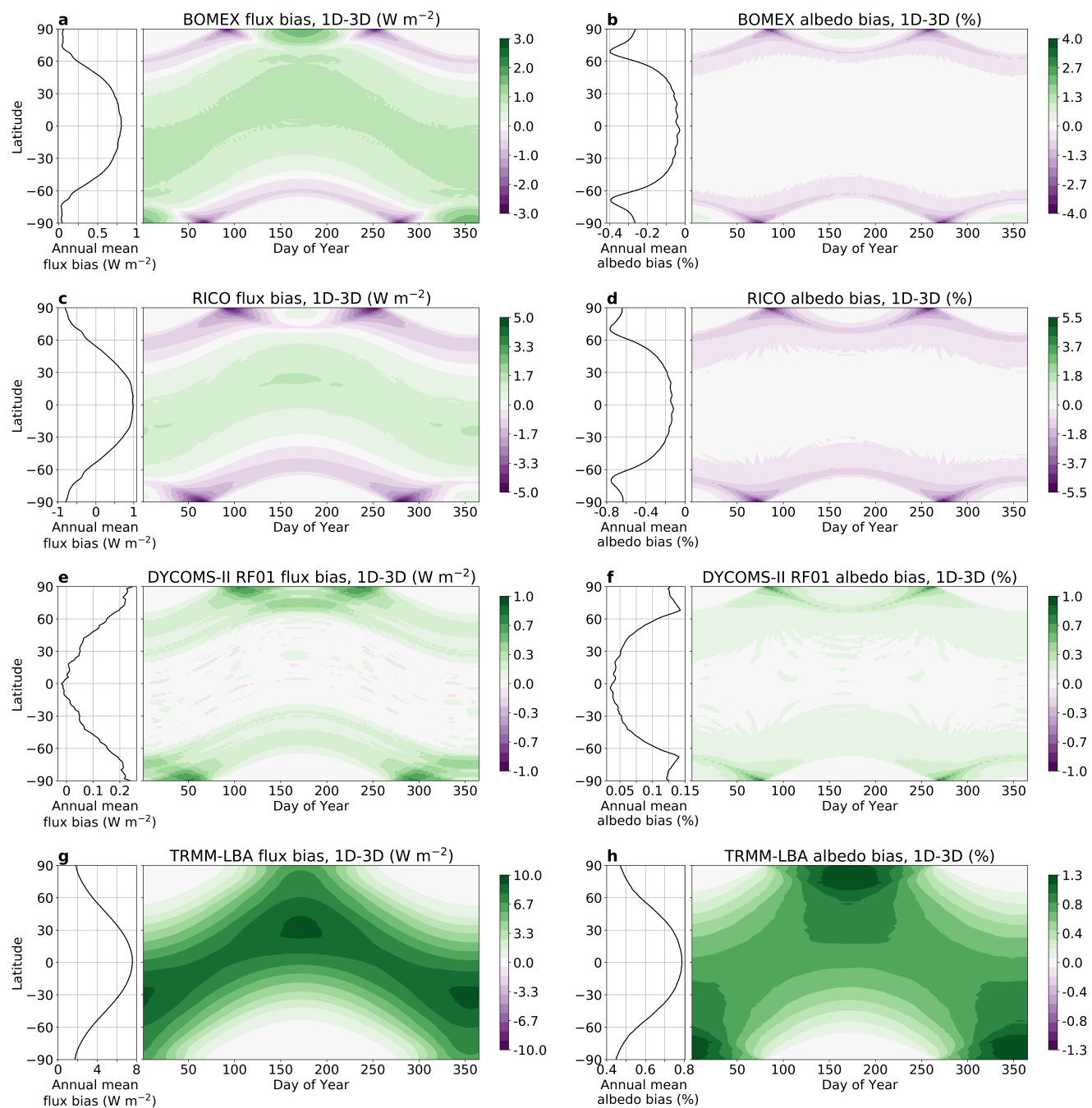

Figure 3. Daily bias (1D-3D) as a function of latitude and day of year assuming the globe is covered by (a-d) ShCu (BOMEX and RICO), (e-f) Sc (DYCOMS-II RF01), and (g-h) Cb (TRMM-LBA). Left column shows flux bias, and right columns shows albedo bias. Inset panels on the left show annual average biases. 

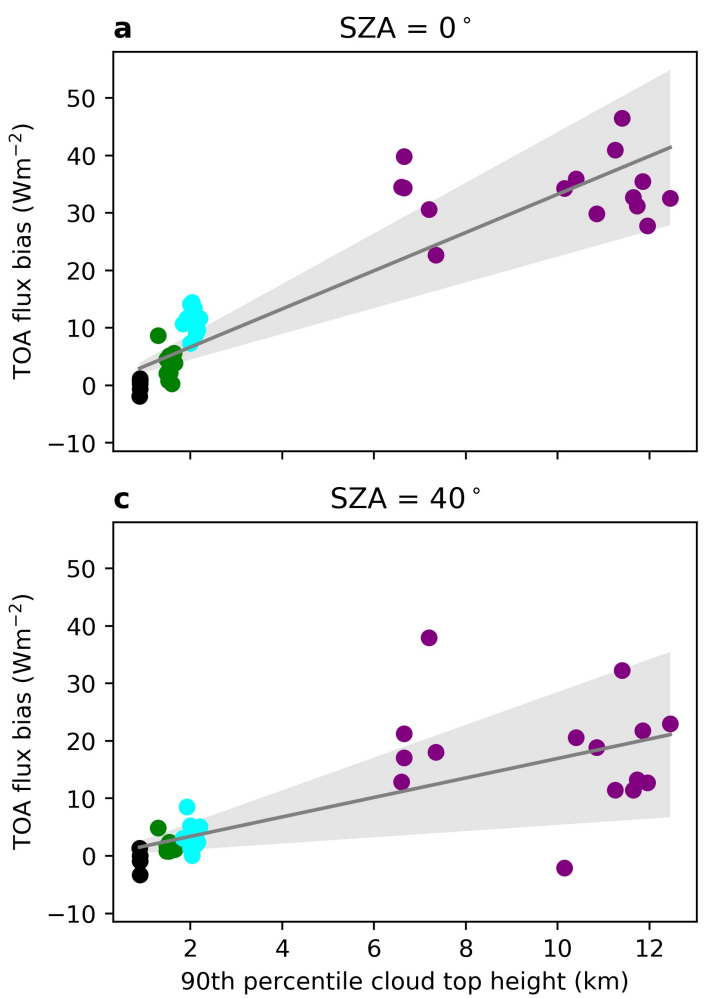
flux bias. b
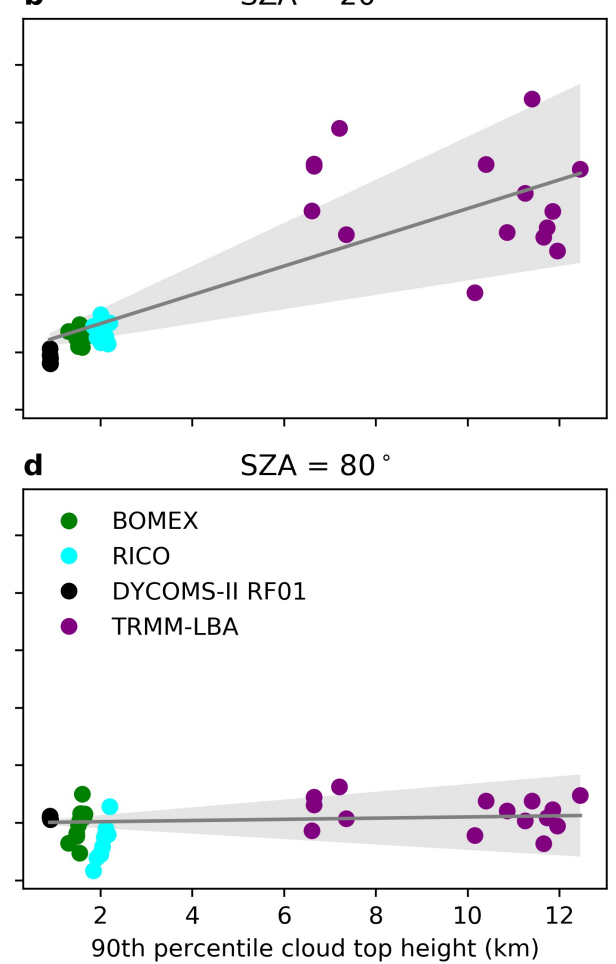

Figure 4. Scatter plot of 90th percentile cloud top height $(\mathrm{CTH})$ from LES domain against flux bias at zenith angles (a) $0^{\circ}$, (b) $20^{\circ}$, (c) $40^{\circ}$, and (d) $80^{\circ}$. LES ensemble members are plotted by color (green, cyan, black, and purple for BOMEX, RICO, DYCOMS-II RF01, and TRMM-LBA, respectively). The grey lines and shaded error bars show the linear fits passing through the origin.

percentile height observed in the LES domain. We choose this metric to exclude small, ephemeral clouds high in the domain. Note that the deviations from the fit of the $\mathrm{Cb}$ clouds suggests that this simple linear model is insufficient. The radiative flux bias depends on more than just $\mathrm{CTH}$, but we use it here as a first approximation to model the 
monthly climatological mean with spatial resolution of $1^{\circ} \times 1^{\circ}$ constructed from measurements during the period $1984-2007$. These data are collected by a suite of weather satellites that are combined into a 3-hourly global gridded product at the D1 level and averaged, including a mean diurnal cycle, into the D2 product we use. The monthly temporal resolution is not inherently an issue for this analysis given that the relationship we use between CTH and flux bias is linear.

To construct the annual-mean flux bias map shown in Figure 5, we first calculate the solar zenith angle for each location on Earth and each hour of the year. Then, we obtain the flux bias given the observed CTH from the linear regression of flux bias against CTH obtained from the LES data at the given zenith angle (Figure 4).
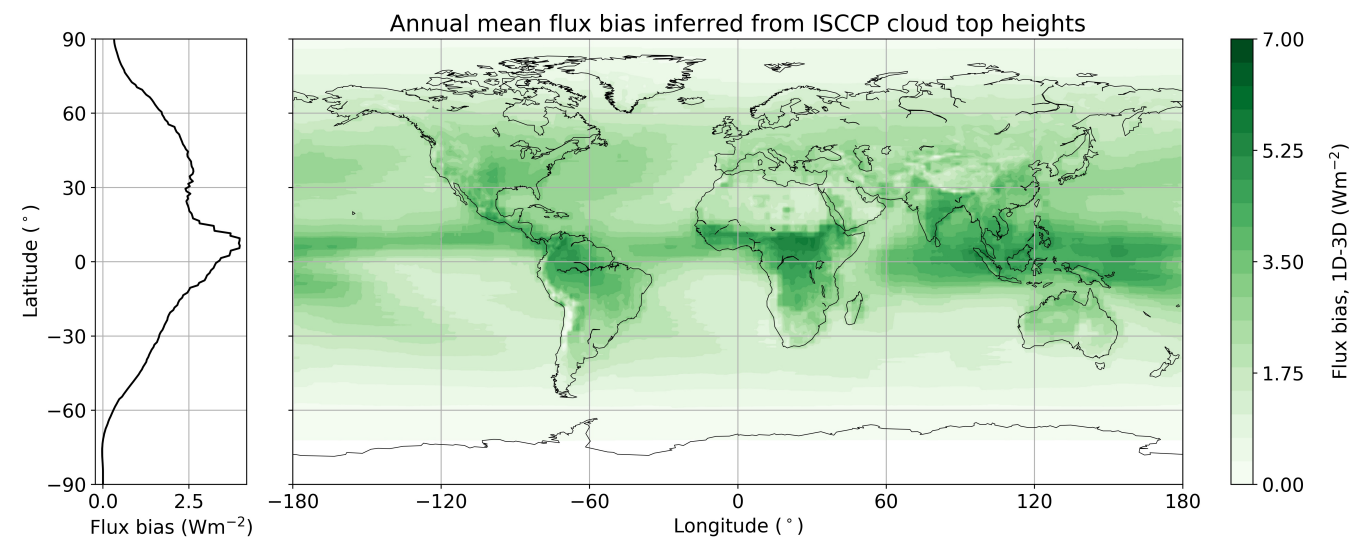

Figure 5. Map of annual mean flux bias inferred from ISCCP CTH. Left panel shows the zonally-averaged flux bias.

The largest bias occurs over the tropics in the ITCZ and over the mid-latitude storm tracks (Figure 5). The large bias occurs where the tallest clouds on Earth exist and where the mean zenith angle is smallest (deep tropics), and also where annual cloud cover is high (storm track regions). Polar regions exhibit a negligible annual-mean flux bias due to the weak incoming solar flux. The maximum flux bias, occurring in the tropics, is around $6 \mathrm{~W} \mathrm{~m}^{-2}$, and the zonal-averaged tropical flux bias is estimated to be $2.7 \pm 0.7 \mathrm{~W} \mathrm{~m}^{-2}$. Our results agree well with those reported in Cole et al. (2005), who employ 2D radiative transfer calculations in a superparameterized ESM with $4 \mathrm{~km}$ horizontal resolution, sufficient to partially resolve deep convective clouds. They found the largest flux bias occurring over the ITCZ region and at small zenith angles where cloud-side illumination 
is important, with a maximum bias of $5 \mathrm{~W} \mathrm{~m}^{-2}$ and tropical zonal-average bias of 1.5 $\mathrm{W} \mathrm{m} \mathrm{m}^{-2}$.

\section{Conclusions}

We have quantified the radiative flux bias that results from making the IPA, using a 3D Monte Carlo radiative transfer scheme applied to LES-generated clouds. The flux bias is assessed across different cloud regimes and solar zenith angles. The bias is largest and positive for deep convective clouds at small zenith angles; however, the albedo bias is largest and negative for shallow cumulus clouds at high zenith angles. The large positive flux bias at small zenith angle for $\mathrm{Cb}$ clouds translates into a seasonal bias that peaks just off the equator in the summer hemisphere, tracking the position of the ITCZ. These results are used alongside observations of the climatological occurrence of clouds to infer the resulting climate impact. The annual-mean global-mean flux bias is $1.6 \pm$ $1.1 \mathrm{~W} \mathrm{~m}^{-2}$. The exact magnitude of the flux bias computed here is minimally sensitive to the spatial resolution of the LES clouds (Supporting Information). We note, however, that even a coarse-resolution LES will resolve cloud morphology with greater detail than an ESM. As shown in Figure 1, the flux and albedo bias across zenith angles is significantly smaller when making the IPA versus the PPA. So the bias from neglect of 3D RT effects in current ESMs is likely larger than the IPA bias which we focused on here.

The flux bias computed here is small compared to the TOA shortwave flux root mean squared error typically in CMIP5 and CMIP6 models, which is on the order of 10 $\mathrm{W} \mathrm{m}^{-2}$ (Zhao et al., 2018; Hourdin et al., 2020). Radiative flux biases attributable to clouds in current ESMs are predominantly due to deficiencies of subgrid-scale dynamical parameterizations that generate cloud cover biases. These biases are distinct from what we documented here, which is the bias that exists purely from neglecting the 3D cloud morphology during the RT computations (i.e., neglecting horizontal photon fluxes when making the IPA). However, as convection parameterizations improve and model resolution increases, the relative contribution of 3D RT effects to the total model error will increase. Additionally, the 3D bias is still large compared to the signal of anthropogenic greenhouse gas emissions, which is on the order of $2.5-3.1 \mathrm{~W} \mathrm{~m}^{-2}$ (Myhre et al., 2013). 
This work is not without caveats. The inferred zenith angle dependence of the flux bias is based on only four LES cases, and therefore does not represent the full diversity of cloud morphologies. There is room for future work considering a larger ensemble of cloud morphologies, which could be generated again by LES or alternatively could be retrieved from satellite observations. Furthermore, the correlation between CTH and flux bias seen in these simulations is very high, but not perfect. There is potential for a more robust mapping from cloud properties to radiative flux bias that could serve as the basis of a new parameterization of 3D RT effects, for inclusion in current ESMs. However, the results highlight the importance of considering the 3D radiative fluxes through clouds for Earth's radiation budget.

\section{Acknowledgments}

C.E.S. acknowledges support from NSF Graduate Research Fellowship under Grant No. DGE-1745301. I.L. is supported by a fellowship from the Resnick Sustainability Institute at Caltech. This research was additionally supported by the generosity of Eric and Wendy Schmidt by recommendation of the Schmidt Futures program and by Mountain Philanthropies. Part of this research was carried out at the Jet Propulsion Laboratory, California Institute of Technology, under a contract with the National Aeronautics and Space Administration.

All code or data used in this paper are freely available online. The LES were run using the PyCLES code (https://climate-dynamics.org/software/\#pycles). The radiative transfer computations were done using the libRadtran code (http://www.libradtran .org). Post-processed LES 3D fields used as input files for libRadtran computations are available in Singer et al. (2020). The ISCCP data were downloaded from the GEWEX database (https://climserv.ipsl.polytechnique.fr/gewexca/).

\section{References}

Ackerman, A. S., VanZanten, M. C., Stevens, B., Savic-Jovcic, V., Bretherton, C. S., Chlond, A., ... Zulauf, M. (2009). Large-eddy simulations of a drizzling, stratocumulus-topped marine boundary layer. Monthly Weather Review, 137(3), 1083-1110. doi: 10.1175/2008MWR2582.1

Barker, H. W. (1994). Solar radiative transfer for wind-sheared cumulus cloud fields. Journal of the Atmospheric Sciences, 51(9), 1141-1156. doi: 

10.1175/1520-0469(1994)051〈1141:SRTFWS $\rangle 2.0 . C O ; 2$

Barker, H. W., Cole, J. N. S., Li, J., Yi, B., \& Yang, P. (2015). Estimation of Errors in Two-Stream Approximations of the Solar Radiative Transfer Equation for Cloudy-Sky Conditions. Journal of the Atmospheric Sciences, 72(11), 4053-4074. doi: 10.1175/JAS-D-15-0033.1

Barker, H. W., Kato, S., \& Wehr, T. $\quad$ (2012). $\quad$ Computation of solar radiative fluxes by 1D and 3D methods using cloudy atmospheres inferred from A-train satellite data. $\quad$ Surveys in Geophysics, 33(3-4), 657-676. doi: 10.1007/s10712-011-9164-9

Barker, H. W., Stephens, G. L., Partain, P. T., Bergman, J. W., Bonnel, B., Campana, K., .. Yang, F. (2003). Assessing 1D Atmospheric Solar Radiative Transfer Models: Interpretation and Handling of Unresolved Clouds. Journal of Climate, 16(16), 2676-2699. doi: 10.1175/1520-0442(2003)016<2676: ADASRT $>2.0 . \mathrm{CO} ; 2$

Bender, F. A.-M., Rodhe, H., Charlson, R. J., Ekman, A. M. L., \& Loeb, N. (2006). 22 views of the global albedo - comparison between 20 GCMs and two satellites. Tellus A: Dynamic Meteorology and Oceanography, 58(3), 320-330. doi: 10.1111/j.1600-0870.2006.00181.x

Blossey, P. N., Bretherton, C. S., Zhang, M., Cheng, A., Endo, S., Heus, T., ... Xu, K.-M. (2013). Marine low cloud sensitivity to an idealized climate change: The CGILS LES intercomparison. Journal of Advances in Modeling Earth Systems, 5(2), 234-258. doi: 10.1002/jame.20025

Cahalan, R., \& Wiscombe, W. (1992). Proceedings of the Second Atmospheric Radiation Measurement (ARM) Science Team Meeting. In Plane-parallel albedo bias (p. 35). Denver, Colorado.

Cahalan, R. F., Ridgway, W., Wiscombe, W. J., Gollmer, S., \& Harshvardhan. (1994). Independent Pixel and Monte Carlo Estimates of Stratocumulus Albedo. Journal of the Atmospheric Sciences, 51(24), 3776-3790. doi: 10.1175/1520-0469(1994)051〈3776:IPAMCE $\rangle 2.0 . C O ; 2$

Cole, J. N. S., Barker, H. W., O'Hirok, W., Clothiaux, E. E., Khairoutdinov, M. F., \& Randall, D. A. (2005). Atmospheric radiative transfer through global arrays of 2D clouds. Geophysical Research Letters, 32(19). doi: 10.1029/2005GL023329 
Emde, C., Buras-Schnell, R., Kylling, A., Mayer, B., Gasteiger, J., Hamann, U., ... Bugliaro, L. (2016). The libRadtran software package for radiative transfer calculations (version 2.0.1). Geoscientific Model Development, 9(5), 1647-1672. doi: 10.5194/gmd-9-1647-2016

Frame, J. W., Petters, J. L., Markowski, P. M., \& Harrington, J. Y. (2009). An application of the tilted independent pixel approximation to cumulonimbus environments. Atmospheric Research, 91, 127-136. ～doi: 10.1016/ j.atmosres.2008.05.005

Gimeno García, S., Trautmann, T., \& Venema, V. (2012). Reduction of radiation biases by incorporating the missing cloud variability by means of downscaling techniques: a study using the 3-D MoCaRT model. Atmospheric Measurement Techniques, 5(9), 2261-2276. doi: 10.5194/amt-5-2261-2012

Grabowski, W. W., Bechtold, P., Cheng, A., Forbes, R., Halliwell, C., Khairoutdinov, M., ... Xu, K.-M. (2006). Daytime convective development over land: A model intercomparison based on LBA observations. Quarterly Journal of the Royal Meteorological Society, 132(615), 317-344. doi: 10.1256/qj.04.147

Gristey, J. J., Feingold, G., Glenn, I. B., Schmidt, K. S., Chen, H., Gristey, J. J., ... Chen, H. (2019). Surface solar irradiance in continental shallow cumulus fields: Observations and large eddy simulation. Journal of the Atmospheric Sciences, 19-0261. doi: 10.1175/JAS-D-19-0261.1

Hinkelman, L. M., Evans, K. F., Clothiaux, E. E., Ackerman, T. P., Stackhouse, P. W., Hinkelman, L. M., .. Jr., P. W. S. (2007). The effect of cumulus cloud field anisotropy on domain-averaged solar fluxes and atmospheric heating rates. Journal of the Atmospheric Sciences, 64(10), 3499-3520. doi: $10.1175 / \mathrm{JAS} 4032.1$

Hogan, R. J., \& Shonk, J. K. P. (2013). Incorporating the Effects of 3D Radiative Transfer in the Presence of Clouds into Two-Stream Multilayer Radiation Schemes. Journal of the Atmospheric Sciences, 70(2), 708-724. doi: 10.1175/JAS-D-12-041.1

Hourdin, F., Rio, C., Grandpeix, J.-Y., Madeleine, J.-B., Cheruy, F., Rochetin, N., ... Ghattas, J. (2020). LMDZ6A: the atmospheric component of the IPSL climate model with improved and better tuned physics. Journal of Advances in Modeling Earth Systems. doi: 10.1029/2019MS001892 
Khairoutdinov, M. F., \& Randall, D. A. (2001). A cloud resolving model as a cloud parameterization in the NCAR Community Climate System Model: Preliminary results. Geophysical Research Letters, 28(18), 3617-3620. doi: 10.1029/2001GL013552

Kooperman, G. J., Pritchard, M. S., Burt, M. A., Branson, M. D., \& Randall, D. A. (2016). Robust effects of cloud superparameterization on simulated daily rainfall intensity statistics across multiple versions of the Community Earth System Model. Journal of Advances in Modeling Earth Systems, 8(1), 140165. doi: 10.1002/2015MS000574

Macke, A., Mitchell, D., \& Bremen, L. (1999). Monte Carlo radiative transfer calculations for inhomogeneous mixed phase clouds. Physics and Chemistry of the Earth, Part B: Hydrology, Oceans and Atmosphere, 24(3), 237-241. doi: 10.1016/S1464-1909(98)00044-6

Marchand, R., Ackerman, T., Smyth, M., \& Rossow, W. B. (2010). $\quad$ A review of cloud top height and optical depth histograms from MISR, ISCCP, and MODIS. Journal of Geophysical Research, 115(D16). doi: 10.1029/2009JD013422

Marshak, A., \& Davis, A. (Eds.). $\quad$ (2005). $\quad 3$ D radiative transfer in cloudy atmospheres. Berlin/Heidelberg: Springer-Verlag. doi: 10.1007/3-540-28519-9

Marshak, A., Davis, A., Wiscombe, W., \& Cahalan, R. (1995). Radiative smoothing in fractal clouds. Journal of Geophysical Research, 100(D12), 26247. doi: 10.1029/95JD02895

Marshak, A., Davis, A., Wiscombe, W., \& Titov, G. (1995). The verisimilitude of the independent pixel approximation used in cloud remote sensing. Remote Sensing of Environment, 52, 71-78. doi: 10.1016/0034-4257(95)00016-T

Mayer, B. (2009). Radiative transfer in the cloudy atmosphere. EPJ Web of Conferences, 1, 75-99. doi: 10.1140/epjconf/e2009-00912-1

Mayer, B., \& Kylling, A. (2005). Technical note: The libRadtran software package for radiative transfer calculations - description and examples of use. Atmospheric Chemistry and Physics, 5(7), 1855-1877. doi: 10.5194/ acp-5-1855-2005

McKee, T. B., \& Cox, S. K. (1974). Scattering of visible radiation by finite clouds. Journal of the Atmospheric Sciences, 31(7), 1885-1892. doi: 
10.1175/1520-0469(1974)031<1885:SOVRBF $\rangle 2.0 . \mathrm{CO} ; 2$

Myhre, G., Shindell, D., Bréon, F.-M., Collins, W., Fuglestvedt, J., Huang, J., ... Zhang, H. (2013). Anthropogenic and Natural Radiative Forcing. In T. Stocker et al. (Eds.), Climate Change 2013: The Physical Science Basis. Contribution of Working Group I to the Fifth Assessment Report of the Intergovernmental Panel on Climate Change (pp. 659-740). Cambridge, United Kingdom and New York, NY, USA: Cambridge University Press.

Okata, M., Nakajima, T., Suzuki, K., Inoue, T., Nakajima, T. Y., \& Okamoto, H. (2017). A study on radiative transfer effects in 3-D cloudy atmosphere using satellite data. Journal of Geophysical Research: Atmospheres, 122(1), 443-468. doi: 10.1002/2016JD025441

Oreopoulos, L., \& Davies, R. (1998). Plane Parallel Albedo Biases from Satellite Observations. Part I: Dependence on Resolution and Other Factors. Journal of Climate, 11 (5), 919-932. doi: 10.1175/1520-0442(1998)011〈0919:PPABFS $\rangle 2.0$ . $\mathrm{CO} ; 2$

Pressel, K. G., Kaul, C. M., Schneider, T., Tan, Z., \& Mishra, S. (2015). Largeeddy simulation in an anelastic framework with closed water and entropy balances. Journal of Advances in Modeling Earth Systems, 7(3), 1425-1456. doi: 10.1002/2015MS000496

Pressel, K. G., Mishra, S., Schneider, T., Kaul, C. M., \& Tan, Z. (2017). Numerics and subgrid-scale modeling in large eddy simulations of stratocumulus clouds. Journal of Advances in Modeling Earth Systems, 9(2), 1342-1365. doi: 10.1002/2016MS000778

Rossow, W., \& Duenas, E. (2004). The International Satellite Cloud Climatology Project (ISCCP) web site: An online resource for research.

Bulletin of the American Meteorological Society, 85(2), 167-176.

doi: $10.1175 /$ BAMS-85-2-167

Rossow, W. B., Schiffer, R. A., Rossow, W. B., \& Schiffer, R. A. (1999). Advances in understanding clouds from ISCCP. Bulletin of the American Meteorological Society, 80 (11), 2261-2287. doi: 10.1175/1520-0477(1999)080〈2261:AIUCFI $>2.0$ . $\mathrm{CO} ; 2$

Schäfer, S. A. K., Hogan, R. J., Klinger, C., Chiu, J. C., \& Mayer, B. (2016). Representing 3-D cloud radiation effects in two-stream schemes: 1. Longwave con- 
siderations and effective cloud edge length. Journal of Geophysical Research: Atmospheres, 121(14), 8567-8582. doi: 10.1002/2016JD024876

Schneider, T., Teixeira, J., Bretherton, C. S., Brient, F., Pressel, K. G., Schär, C., \& Siebesma, A. P. (2017). Climate goals and computing the future of clouds. $\quad$ Nature Climate Change opinion $\&$ comment, 7(1), 3-5. doi: 10.1038/nclimate3190

Siebesma, A. P., Bretherton, C. S., Brown, A., Chlond, A., Cuxart, J., Duynkerke, P. G., ... Stevens, D. E. (2003). A large eddy simulation intercomparison study of shallow cumulus convection. Journal of the Atmospheric Sciences, 60 (10), 1201-1219. doi: 10.1175/1520-0469(2003)60〈1201:ALESIS $\rangle 2.0 . C O ; 2$

Singer, C., Lopez-Gomez, I., Zhang, X., \& Schneider, T. (2020). Data for "Topof-atmosphere albedo bias from neglecting three-dimensional radiative transfer through clouds". CaltechDATA. doi: 10.22002/D1.1637

Stephens, G. L., O’Brien, D., Webster, P. J., Pilewski, P., Kato, S., \& Li, J.-l. (2015). The albedo of Earth. Reviews of Geophysics, 53(1), 141-163. doi: $10.1002 / 2014 R G 000449$

Stevens, B., Moeng, C.-H., Ackerman, A. S., Bretherton, C. S., Chlond, A., de Roode, S., ... Zhu, P. (2005). Evaluation of large-eddy simulations via observations of nocturnal marine stratocumulus. Monthly Weather Review, 133(6), 1443-1462. doi: 10.1175/MWR2930.1

Stubenrauch, C., Rossow, W., \& Kinne, S. (2012). Assessment of global cloud data sets from satellites: A project of the world climate research programme Global Energy and Water Cycle Experiment (GEWEX) Radiation Panel (Tech. Rep. No. 23).

Stubenrauch, C. J., Rossow, W. B., Kinne, S., Ackerman, S., Cesana, G., Chepfer, H., ... Zhao, G. (2013). Assessment of clobal cloud datasets from satellites: Project and database initiated by the GEWEX radiation panel. Bulletin of the American Meteorological Society, 94 (7), 1031-1049. doi: 10.1175/BAMS-D-12-00117.1

Tompkins, A. M., \& Di Giuseppe, F. (2007). Generalizing Cloud Overlap Treatment to Include Solar Zenith Angle Effects on Cloud Geometry. Journal of the Atmospheric Sciences, 64(6), 2116-2125. doi: 10.1175/JAS3925.1

Tompkins, A. M., \& Di Giuseppe, F. (2015). An Interpretation of Cloud Overlap 
Statistics. Journal of the Atmospheric Sciences, 72(8), 2877-2889. doi: 10 $.1175 / J A S-D-14-0278.1$

vanZanten, M. C., Stevens, B., Nuijens, L., Siebesma, A. P., Ackerman, A. S., Burnet, F., ... Wyszogrodzki, A. (2011). Controls on precipitation and cloudiness in simulations of trade-wind cumulus as observed during RICO. Journal of Advances in Modeling Earth Systems, 3(2). doi: 10.1029/2011MS000056

Várnai, T., \& Davies, R. (1999). Effects of cloud heterogeneities on shortwave radiation: Comparison of cloud-top variability and internal heterogeneity. Journal of the Atmospheric Sciences, 56(24), 4206-4224. doi: 10.1175/1520-0469(1999) 056<4206:EOCHOS $>2.0 . \mathrm{CO} ; 2$

Veerman, M. A., Pedruzo-Bagazgoitia, X., Jakub, F., Vilà-Guerau de Arellano, J., \& Heerwaarden, C. C. (2020). Three-Dimensional Radiative Effects By Shallow Cumulus Clouds on Dynamic Heterogeneities Over a Vegetated Surface. Journal of Advances in Modeling Earth Systems, 12(7). doi: 10.1029/2019MS001990

Villefranque, N., Fournier, R., Couvreux, F., Blanco, S., Cornet, C., Eymet, V., ... Tregan, J. (2019). A A Path-Tracing Monte Carlo Library for 3-D Radiative Transfer in Highly Resolved Cloudy Atmospheres. Journal of Advances in Modeling Earth Systems, 11(8), 2449-2473. doi: 10.1029/2018MS001602

Voigt, A., Stevens, B., Bader, J., \& Mauritsen, T. (2013). The observed hemispheric symmetry in reflected shortwave irradiance. J. Climate, 26, 468-477. doi: https://doi.org/10.1175/JCLI-D-12-00132.1

Wissmeier, U., Buras, R., \& Mayer, B. $\quad$ (2013). $\quad$ paNTICA: A fast 3D radiative transfer scheme to calculate surface solar irradiance for NWP and LES models. Journal of Applied Meteorology and Climatology, 52(8), 1698-1715. doi: 10.1175/JAMC-D-12-0227.1

Wood, N. B., Gabriel, P. M., Stephens, G. L., Wood, N. B., Gabriel, P. M., \& Stephens, G. L. (2005). An Assessment of the Parameterization of Subgrid-Scale Cloud Effects on Radiative Transfer. Part II: Horizontal Inhomogeneity. Journal of the Atmospheric Sciences, 62(8), 2895-2909. doi: 10.1175/JAS3498.1

Wyser, K. (1998). The effective radius in ice clouds. Journal of Climate, 11(7), 1793-1802. doi: 10.1175/1520-0442(1998)011〈1793:TERIIC〉2.0.CO;2 
521

522

523

524

525

Zhao, M., Golaz, J.-C., Held, I. M., Guo, H., Balaji, V., Benson, R., ... Xiang, B.

(2018). The GFDL Global Atmosphere and Land Model AM4.0/LM4.0:

2. Model Description, Sensitivity Studies, and Tuning Strategies.

Jour-

nal of Advances in Modeling Earth Systems, 10(3), 735-769. ～doi: 10.1002/

2017MS001209 